\title{
A COMPARISON OF WAVELET-BASED AND RIDGELET- BASED TEXTURE CLASSIFICATION OF TISSUES IN COMPUTED TOMOGRAPHY
}

\author{
Lindsay Semler \\ Lucia Dettori \\ Intelligent Multimedia Processing Laboratory \\ School of Computer Scienve, Telecommunications and Information Systems \\ DePaul University, Chicago IL, 60604, USA \\ lsemler@cs.depaul.edu \\ ldettori@cs.depaul.edu
}

Keywords: $\quad$ Multi-Resolution Analysis, Texture Classification, Wavelet, Ridgelet, Computed Tomography

\begin{abstract}
The research presented in this article is aimed at developing an automated imaging system for classification of tissues in medical images obtained from Computed Tomography (CT) scans. The article focuses on using multi-resolution texture analysis, specifically: the Haar wavelet, Daubechies wavelet, Coiflet wavelet, and the ridgelet. The algorithm consists of two steps: automatic extraction of the most discriminative texture features of regions of interest and creation of a classifier that automatically identifies the various tissues. The classification step is implemented using a cross-validation Classification and Regression Tree approach. A comparison of wavelet-based and ridgelet-based algorithms is presented. Tests on a large set of chest and abdomen CT images indicate that, among the three wavelet-based algorithms, the one using texture features derived from the Haar wavelet transform clearly outperforms the one based on Daubechies and Coiflet transform. The tests also show that the ridgelet-based algorithm is significantly more effective and that texture features based on the ridgelet transform are better suited for texture classification in CT medical images.
\end{abstract}

\section{INTRODUCTION}

The research presented in this article is part of an ongoing project (Xu et al. 2005), (Channin et al. 2004), and (Semler, Dettori, \& Furst 2005) aimed at developing an automated imaging system for classification of tissues in medical images obtained by Computed Tomography (CT) scans. Classification of human organs in CT scans using shape or grey level information is particularly challenging due to the changing shape of organs in a stack of slices in 3D medical images and the grey level intensity overlap in soft tissues. However, healthy organs are expected to have a consistent texture within tissues across multiple slices. This research focuses on using multi-resolution texture analysis for the classification of tissues from normal chest and abdomen CT scans. The approach consists of two steps: extraction of the most discriminative texture features of regions of interest and creation of a classifier that automatically identifies the various tissues. Four forms of multi-resolution analysis were carried on and texture features vectors were created from image transformations based on: the Haar wavelet, the Daubechies wavelet, the Coiflet wavelet, and the ridgelet. The classification step is implemented through a decision tree classifier based on the cross-validation Classification and Regression Tree (C\&RT) approach. Multi-resolution analysis has been successfully used in image processing, and a number of applications to texture classification have been proposed over the past few years. Several studies have investigated the discriminating power of wavelet-based features applied to various domains, examples can be found in (Dara \& Watsuji 2003) and (Mulcahy 1997). Recently, the finite 
ridgelet transform has emerged as a new multiresolution analysis tool. Applications of ridgelet transforms to image contrast enhancement and image denoising have been developed in recent years as in (Do, \& Vetterli 2003), however, to the authors' knowledge, applications to texture classification have only been investigated in the context of natural images (LeBorgne \& O'Connor 2005).

Texture is a commonly used feature in the analysis and interpretation of images. It can be characterized by a set of local statistical properties of the pixel grey level intensity. Statistical, structural, or spectral techniques commonly used are: wavelets, run-length statistics, spectral measures, fractal dimensions, statistical moments, and co-occurrence matrices.

The discrete wavelet transform decomposes the image into several directional details obtaining lowpass bands that capture horizontal, vertical and diagonal activity. First and second order statistics of the wavelet detail coefficients provide texture descriptors that can discriminate contrasting intensity properties spatially distributed throughout the image, according to various levels of resolution. Wavelets have been an area of research in many texture classification applications and have been useful in capturing texture information and edge detection in natural images (Li, Jun 2003), such as detecting the vertical outline of a skyscraper. However, they are not able to capture enough directional information in noisy images, such as medical CT scans.

A better approach to texture classification for this type of images is to apply a ridgelet transform instead of a Wavelet transform. Ridgelets, like wavelets, capture directional information of an image, however, they are not limited to vertical, horizontal, and diagonal directions. Structural information derived from the ridgelet transform of an image is based on multiple radial directions in the frequency domain. For ridgelets, first order statistics can be calculated on the directional detail coefficients, providing texture descriptors that can be used in the classification of texture. Our tests confirm that the multi-directional capabilities of the ridgelet transform provide better texture information and prove to be more effective in the texture classification in medical images.

The article is organized as follows. Section 2 describes the data set, the wavelet and ridgelet transforms and the texture feature extraction process. The classification algorithm is detailed in Section 3. Tests and a comparison of wavelet-based and ridgelet-based features are presented in Section 4.

\section{TEXTURE FEATURES}

The texture classification algorithm proposed in this article consists of four main steps: segmentation of regions of interest (organs), application of the discrete wavelet or ridgelet transform, extraction of texture features, and creation of a classifier. In this article, we analyze and compare texture classification techniques based on four different multi-resolution approaches: Haar $(\mathrm{H})$ wavelet, Daubechies 4 (D4) wavelet, Coiflet (C6) wavelet, and the ridgelet.

A wavelet is a mathematical function that filters a signal or an image with a series of averaging and differencing calculations see for example (Mulcahy 1997). Wavelets are typically used in image decomposition and compression. Wavelets can be calculated according to various levels of resolution (or blurring) depending on how many levels of averages are calculated. They are sensitive to the spatial distribution of grey level pixels, but are also able to differentiate and preserve details at various scales or resolutions.

The ridgelet transform is an application of a multiresolution wavelet to a radon transform. A radon transform is able to provide directional information in the frequency domain. Thus, ridgelets capture several directions, in addition to the horizontal, vertical and diagonal offered by the wavelet. The ridgelet gives rotation invariant structural information on multiple directions and scales.

\subsection{The Data Set}

The texture classification algorithms were tested on 3D data extracted from two normal chest and abdomen CT studies from Northwestern Memorial Hospital. The data consisted of 340 2D DICOM consecutives slices, each slice being 512 x 512 and having 12-bit grey level resolution. Using an Active Contour Models ("Snake") algorithm, five organs were segmented from the initial data: heart, liver, spleen, kidney, and backbone ( $\mathrm{Xu}$ et al. 2005). The segmentation process generated 140 Backbone slices, 52 Heart, 58 Liver, 54 Kidney, and 40 Spleen.

Both wavelets and ridgelets are extremely sensitive to contrast in the grey level intensity, therefore, in order to use wavelet-based or ridgeletbased texture description it was necessary to eliminate all background pixels to avoid mistaking the edge between the artificial background and the organ as a texture feature. Each slice was therefore further cropped, and only square sub-images fully contained in the interior of the segmented area were 
generated. These images were of sizes $31 \times 31$ (for ridgelets) or $32 \times 32$ (for wavelets), resulting in 2,091 slices of "pure" single-organ tissue (363 Backbone, 446 Heart, 506 Liver, 411 Kidney, 364 Spleen). These images were cropped to the respective size because of the requirements of an image of size $2^{\mathrm{n}}$ for wavelets and a prime image size for ridgelets.

\subsection{Feature Extraction}

Once the medical images have been segmented, the wavelet and ridgelet discrete transforms are applied. Several texture features are then extracted from the wavelet and ridgelet coefficients generated by these transforms. First, the three different families of wavelets were investigated to determine which would yield a higher discriminating power. Haar, Daubechies and Coiflet wavelet filters were applied to each of the images, using two levels of resolution. At each resolution level, three detail coefficient matrices were calculated capturing the vertical, horizontal and diagonal structures of the image.

The following first order statistics were calculated on each of the directional matrices: Mean and Standard Deviation. Also calculated from these matrices were 4-directional co-occurrence matrices on which the following second order statistics were calculated: Energy, Entropy, Contrast, Homogeneity Sum-mean, Variance, Maximum Probability, Inverse Difference Moment, and Cluster Tendency (Haralick, Shanmugame, \& Dinstein 1973). This generated a 264-element texture descriptor vector per image. To avoid problems of overfitting for the decision trees the resulting feature vector was reduced to 22 features (using only two levels of resolution and averaging over wavelet details and co-occurrence directions). Further details on feature vector reduction and more in-depth analysis of the various wavelet-based texture features are provided in (Semler, Dettori, \& Furst 2005).

The Finite Ridgelet Transform as presented in (Do \& Vetterli 2003), was also applied. This was computed by: first calculating a discrete radon transform, and then applying a one-dimensional wavelet transform. The radon transform was computed by: first calculating the 2-dimensional fast Fourier transform of the image, and then applying a 1-dimensional inverse Fourier transform on each of the 32 radial directions of the radon projection. A one-dimensional Haar wavelet was applied to each of the radial directions, for two levels of resolution. The following texture descriptors were then calculated for each radial direction and resolution level of the wavelet details: mean, standard deviation, energy and entropy. Entropy texture descriptors were determined to yield the highest discriminating power; further details are presented in (Semler, Dettori \& Kerr 2006). Several different combinations of resolution levels were also investigated, and two levels of resolution were determined best for both ridgelets and wavelets. The numbers of features extracted were limited since each descriptor is calculated over two resolution levels and for 32 directions, yielding 64 descriptors.

Although the ridgelet-based features contain more descriptors, it should not be assumed they would perform better than the wavelet-based features because of the increase in number of descriptors. In (Semler, Dettori, \& Furst 2005), it was found that a wavelet-based feature vector of 33 descriptors outperformed another same-family wavelet-based feature vector of 99 descriptors.

\section{TEXTURE CLASSIFICATION}

The classification step was carried out using a decision tree classifier based on the Classification and Regression Tree (C\&RT) approach (Channin et al. 2004). A decision tree predicts the class of an object (organ) from values of predictor variables (texture descriptors). The most relevant texture descriptors are found for each specific organ, and based on those selected descriptors, a set of decision rules are generated. These set of rules are then used for the classification of the each region. Using the C\&RT cross-validation approach, each tree's parameter was optimized, including depth of tree, number of parent nodes, and number of child nodes.

To evaluate the performance of each classifier, specificity, sensitivity, precision, and accuracy rates were calculated from each of the misclassification matrices.

A misclassification matrix is a table that lists each organ and its true positives, true negatives, false positives and false negatives. The number of true positives is the number of organs that are correctly classified as that organ. The number of true negatives is the number of other organs that are correctly classified as other organs. The number of false positives is the number of organs that are incorrectly classified as that organ. The number of false negatives is the number of organs that are incorrectly classified as other organ. From the misclassification matrix specificity, sensitivity, precision, and accuracy statistics were computed. 
Table 1: Measures of classification performance

\begin{tabular}{|l|l|}
\hline Measure & Definition \\
\hline Sensitivity & True Positive / Total Positive \\
\hline Specificity & True Negative / Total Negatives \\
\hline Precision & True Positive / (True Positive + False Positives) \\
\hline Accuracy & (True Positives + True Negatives) / Total Sample \\
\hline
\end{tabular}

Specificity measures the accuracy among positive instances, and is calculated by dividing the true negatives by the number of all other organ slices. Sensitivity measures the accuracy among negative instances, and is calculated by dividing the number of true positives by the total number of that specific organ slices. Precision measures show how consistent the results can be reproduced. Accuracy reflects the overall correctness of the classifier, and is calculated by adding the true positives and negatives together and dividing by the entire number of organ slices.

\section{WAVELET - RIDGELET COMPARISON}

Tables 2-5 in the Appendix show a comparison of accuracy, precision, specificity, and sensitivity results, for each tissue of interest for the three wavelet-based texture features and the ridgelet-based texture features respectively. Within all the wavelets, the Haar wavelet outperformed all others for most organs and performance measures. The only exception is the backbone, for which the Daubechies and Coiflet wavelets produce slightly better results. The performance for the Haar-based descriptors in all other organs was significantly higher, thus indicating that these descriptors yield the highest discriminating power among the wavelet-based features.

The results also show that the ridgelet-based texture features outperform all wavelet-based descriptors. Accuracy rates for Wavelet-based texture descriptors range between 85 - 93\%, while ridgelet-based accuracy rates are in the $91-97 \%$ range. Precision rates for the wavelets are between $55-91 \%$, compared to $73-93 \%$ for ridgelets. Specificity rates for the wavelets are in the $82-97 \%$ range, while specificity for the ridgelet descriptors is in the $92-98 \%$ range. Furthermore, sensitivity rates for the wavelets are in the $35-87 \%$ range, whereas ridgelets are between $72-94 \%$. The lower bound of the sensitivity range for wavelets is due to the poor performance of those descriptors (especially Coiflets and Daubechies) for Heart and Spleen. The texture of the images for these two organs is quite similar and the classifier often mistakes the two organs for one another. Further investigation is needed to determine the underlying cause for the poor performance of the Heart and Spleen.

Overall, the ridgelet-based descriptors have significantly higher performance measures, with accuracy rates approximately four percent higher than any other feature set for all individual organs. This was expected due to the fact that the ridgelet transform is able to capture multi-directional features, as opposed to the wavelet transform which focuses mainly on horizontal, vertical, and diagonal features, which are not dominant in medical CT scan images. One of the limitations of using ridgeletbased descriptors is the fact that ridgelets are most effective in detecting linear radial structures, which are not the main component of medical images. A recent extension of ridgelets is the curvelet transform. Curvelets have been proven to be particularly effective at detecting image activity along curves instead of radial directions (Starck Donoho \& Candes 1999). We are currently investigating the use of curvelet-based texture descriptors and we expect this to further improve the ability of our classifier to successfully classify each tissue sample.

\section{REFERENCES}

[1] Xu, D., Lee, J., Raicu, D.S., Furst, J.D., \& Channin, D. (2005). Texture Classification of Normal Tissues in Computed Tomography. The 2005 Annual Meeting of the Society for Computer Applications in Radiology.

[2] Channin, D., Raicu, D.S., Furst, J.D., Xu, D.H., Lilly, L., \& Limpsangsri, C. (2004). Classification of Tissues in Computed Tomography using Decision Trees. Poster and Demo. The $90^{\text {th }}$ Scientific Assembly and Annual Meeting of Radiology Society of North America.

[3] Semler, L., Dettori, L., \& Furst, J. (2005). WaveletBased Texture Classification of Tissues in Computed Tomography. Proceedings of the 18th IEEE International Symposium on Computer-Based Medical Systems, 265-270.

[4] Do, M.N., \& Vetterli, M. (2003) The Finite Ridgelet Transform for Image Representation. IEEE Transactions on Image Processing, 12, 16 - 28.

[5] LeBorgne, H.L., \& O'Connor, N. (2005). Natural Scene Classification and Retrieval Using Ridgeletbased Image Signatures. Advanced Concepts for Intelligent Vision Systems, 20-23.

[6] Starck, J.L., Donoho, D.L., \& Candes, E.J. (1999). Astronomical Image Representation by the Curvelet Transform. Astronomy \&Astrophysics, 398, 785-800. 
[7] Semler, L., Dettori, L., \& Kerr, B. (2006). RidgeletBased Texture Classification in Computed Tomography, submitted to IEEE Computer Society Conference on Computer Vision and Pattern Recognition.

[8] Dara, B \& Watsuji, N. (2003). Using Wavelets for Texture Classification. IJCI Proceedings of International Conference on Signal Processing, ISN 1304-2386.

[9] Mulcahy, C. (1997). Image Compression Using the Haar Wavelet Transform. Spelman Science \& Math Journa1, 1, 22-31.
[10] Li, Jun. (2003). A Wavelet Approach to Edge Detection, Master of Science thesis, Sam Houston State University. Huntsville, Texas.

[11] Do, M., \& Vetterli, M. (2003). Image Denoising Using Orthonormal Finite Ridgelet Transform. Proceedings of SPIE: Wavelet Applications in Signal and Image Processing, 4119, 831-842.

[12] Haralick, R. M., Shanmugam, D., \& Dinstein, I. (1973). Texture Features for Image Classification. IEEE Transactions on Systems, Man, and Cybernetics, $3,6,610-621$

\section{APPENDIX}

Table 2: Wavelet-Ridgelet accuracy rates comparison

\begin{tabular}{|lcccccc|}
\hline & \multicolumn{9}{c}{ Accuracy } \\
Feature Set & Backbone & Heart & Liver & Kidney & Spleen & Average \\
Haar & 93.7 & 85.0 & 88.6 & 92.8 & 89.5 & 89.9 \\
Daubechies & 93.6 & 84.0 & 88.0 & 83.6 & 88.2 & 87.5 \\
Coiflets & 93.1 & 85.3 & 88.3 & 85.8 & 88.6 & 88.2 \\
Ridgelet & 97.3 & 93.6 & 92.7 & 92.7 & 91.7 & 93.6 \\
\hline
\end{tabular}

Table 3: Wavelet-Ridgelet precision rates comparison

\begin{tabular}{|lccccccc|}
\hline & \multicolumn{9}{c}{ Precision } & Kackbone & Heart & Liver & Kidney & Spleen & Average \\
Haar & 82.6 & 67.0 & 69.9 & 82.6 & 69.7 & 74.4 \\
Daubechies & 91.6 & 57.4 & 55.7 & 64.9 & 64.3 & 66.8 \\
Coiflets & 90.7 & 58.9 & 56.7 & 70.6 & 70.8 & 69.5 \\
Ridgelet & 93.5 & 90.8 & 79.4 & 88.5 & 72.9 & 85.0 \\
\hline
\end{tabular}

Table 4: Wavelet-Ridgelet specificity rates comparison

\begin{tabular}{|lccccccc|}
\hline & \multicolumn{9}{c}{ Specificity } \\
Feature Set & Backbone & Heart & Liver & Kidney & Spleen & Average \\
Haar & 96.1 & 92.1 & 91.4 & 94.4 & 94.3 & 93.7 \\
Daubechies & 97.3 & 91.8 & 92.0 & 82.9 & 96.2 & 92.0 \\
Coiflets & 96.8 & 89.4 & 92.2 & 87.4 & 97.6 & 92.7 \\
Ridgelet & 98.7 & 97.9 & 92.3 & 97.67 & 93.4 & 96.0 \\
\hline
\end{tabular}

Table 5: Wavelet-Ridgelet sensitivity rates comparison

\begin{tabular}{|lcccccc|}
\hline & \multicolumn{7}{c|}{ Sensitivity } & Kackbone & Heart & Liver & Kidney & Spleen & Average \\
Haar & 82.6 & 59.0 & 77.7 & 87.3 & 65.5 & 74.4 \\
Daubechies & 83.5 & 49.1 & 63.2 & 85.4 & 40.2 & 64.2 \\
Coiflets & 85.9 & 67.1 & 64.3 & 81.6 & 35.2 & 66.8 \\
Ridgelet & 90.9 & 77.8 & 94.2 & 72.5 & 83.8 & 83.8 \\
\hline
\end{tabular}

